\title{
Intergroup Rhabdomyosarcoma Group I
}

National Cancer Institute

\section{Source}

National Cancer Institute. Intergroup Rhabdomyosarcoma Group I. NCI Thesaurus. Code C148012.

Localized disease, excised. 